\title{
Electronic Health Data Predict Outcomes After Aneurysmal Subarachnoid Hemorrhage
}

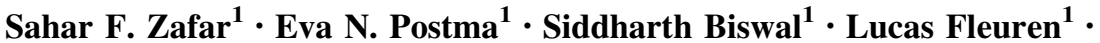 \\ Emily J. Boyle ${ }^{1} \cdot$ Sophia Bechek $^{1} \cdot$ Kathryn O'Connor $^{1} \cdot$ Apeksha Shenoy $^{1}$. \\ Durga Jonnalagadda $^{1} \cdot$ Jennifer Kim $^{1} \cdot$ Mouhsin S. Shafi $^{2} \cdot$ Aman B. Patel $^{3}$. \\ Eric S. Rosenthal ${ }^{1} \cdot$ M. Brandon Westover ${ }^{1}$
}

Published online: 5 October 2017

(C) Springer Science+Business Media, LLC 2017

\begin{abstract}
Backgroud Using electronic health data, we sought to identify clinical and physiological parameters that in combination predict neurologic outcomes after aneurysmal subarachnoid hemorrhage (aSAH).

Methods We conducted a single-center retrospective cohort study of patients admitted with aSAH between 2011 and 2016. A set of 473 predictor variables was evaluated. Our outcome measure was discharge Glasgow Outcome Scale (GOS). For laboratory and physiological data, we computed the minimum, maximum, median, and variance for the first three admission days. We created a penalized logistic regression model to determine predictors of outcome and a multivariate multilevel prediction model to predict poor (GOS 1-2), intermediate (GOS 3), or good (GOS 4-5) outcomes.

Results One hundred and fifty-three patients met inclusion criteria; most were discharged with a GOS of 3. Multivariate analysis predictors of mortality (AUC 0.9198) included APACHE II score, Glasgow Come Scale (GCS), white blood
\end{abstract}

Sahar F. Zafar and Eva N. Postma have contributed equally to the work.

Electronic supplementary material The online version of this article (doi:10.1007/s12028-017-0466-8) contains supplementary material, which is available to authorized users.

Sahar F. Zafar

sfzafar@mgh.harvard.edu

1 Department of Neurology, Lunder 6 Neurosciences Intensive Care Unit, Massachusetts General Hospital, 55 Fruit Street, Boston, MA 02114, USA

2 Department of Neurology, Beth Israel Deaconess Medical Center, Boston, MA, USA

3 Department of Neurosurgery, Massachusetts General Hospital, Boston, MA, USA cell (WBC) count, mean arterial pressure, variance of serum glucose, intracranial pressure (ICP), and serum sodium. Predictors of death/dependence versus independence (GOS 4-5)(AUC 0.9456) were levetiracetam, mechanical ventilation, WBC count, heart rate, ICP variance, GCS, APACHE II, and epileptiform discharges. The multiclass prediction model selected GCS, admission APACHE II, periodic discharges, lacosamide, and rebleeding as significant predictors; model performance exceeded $80 \%$ accuracy in predicting poor or good outcome and exceeded $70 \%$ accuracy for predicting intermediate outcome.

Conclusions Variance in early physiologic data can impact patient outcomes and may serve as targets for early goaldirected therapy. Electronically retrievable features such as ICP, glucose levels, and electroencephalography patterns should be considered in disease severity and risk stratification scores.

Keywords Subarachnoid hemorrhage .

Predictive analytics - Neurologic outcomes .

Machine learning $\cdot$ EEG

\section{Introduction}

Patients who initially survive aneurysmal subarachnoid hemorrhage $(\mathrm{aSAH})$ are at risk of further neurological complications, including delayed cerebral ischemia (DCI), hydrocephalus, rebleeding, and seizures [1]. In addition, medical complications such as cardiac injury and healthcare-associated infections (HAI) can further impact immediate outcomes [2]. Quantifying the degree to which these complications affect outcomes is important for prognostication, and identifying high-impact modifiable factors. Many aSAH severity scores rely primarily on 
initial imaging and the neurological examination [1, 3, 4], which is subject to neurosurgical procedures, sedatives, or paralytics. Though early physiologic data are incorporated in more recent scores $[5,6]$, physiologic data more specific to the nervous system and modifiable complications beyond the early phase are not included.

With the electronic health record (EHR), large amounts of data are available, providing an opportunity to more accurately predict outcomes. Using data-driven predictive modeling, we sought to identify reproducible clinical parameters during hospitalization that may impact discharge outcomes and subsequent rehabilitation potential, serve as potential targets for intervention, and that have not been included in prior severity scores.

\section{Methods}

\section{Patient Cohort}

We performed a retrospective study of patients from the Massachusetts General Hospital aSAH database admitted between September 2011 and February 2016, after institutional review board approval. The database includes patients with high-grade SAH $(\geq$ HH3F3) and who undergo continuous electroencephalogram (EEG) or multimodality monitoring. We included cases with an identified aneurysm and excluded non-aneurysmal and traumatic SAH and cases caused by other vascular malformations. All patients routinely underwent computed tomography angiography and conventional angiography.

Our primary objective was to identify modifiable complications that can impact the hospital course and subsequent rehabilitation potential. Our primary outcome measure was discharge Glasgow Outcome Scale (GOS); GOS 1: death; GOS 2: vegetative state; GOS 3: severe disability; GOS 4: moderate disability; GOS 5: good recovery [7]. Two raters (SFZ and ENP) independently abstracted and adjudicated GOS from physician and physical therapy examinations at discharge and initial rehabilitation facility examinations. Both GOS and GOS extended have been used as discharge outcome measures in prior studies [8-10]. We chose GOS due to its simple categories and ease of ascertainment. To ensure sufficient numbers and data balance for model fitting, we categorized outcomes as: poor (GOS 1-2), intermediate (GOS 3), and good (GOS 4-5).

\section{Candidate Predictors for Outcomes}

We collected demographic and clinical variables from the EHR. A full list of predictor variables is provided in supplementary material (Table e-1) and included admission Glasgow Coma Scale (GCS), APACHE II (Acute Physiology and Chronic Health Evaluation II) score, EEG findings, anti-epileptic drugs (AED), mechanical ventilation, and HAIs. We chose variables based on existing SAH disease severity scores and additional variables that reflect neurological and medical complications.

EEG findings were defined using the American Clinical Neurophysiology Society Nomenclature: sporadic epileptiform discharges, periodic patterns, rhythmic delta activity, and seizures [11]. The institution's EEG protocol for DCI detection recommends 10 days of monitoring for high-grade $(\geq \mathrm{HH} 3 \mathrm{~F} 3$ ) patients. In addition, patients with concern for subclinical seizures are monitored as indicated. The standard practice during the study period was to discontinue AED prophylaxis within $24 \mathrm{~h}$ of aneurysm coiling or within 7 days of clipping. We included AEDs in our analysis, if continued beyond 7 days. Our primary indications for AED continuation are: clinical or electrographic seizures, and scalp or depth ictal-inter-ictal continuum (IIC) patterns or epileptiform discharges at the treating physicians discretion. Levetiracetam is the typical first-line AED. Additional or alternate AEDs, frequently phenytoin and lacosamide, are used for refractory seizures or persistent IIC patterns at the treating physician's discretion.

We chose APACHE II, instead of APACHE III, as it is included in the Functional Recovery Expected after Subarachnoid Hemorrhage (FRESH) score, allowing for more direct comparison. HAIs were confirmed by positive cultures, or radiographic and clinical evidence of respiratory tract infections. Laboratory data (sodium, potassium, glucose, and white blood cell (WBC) count) and physiological data (heart rate, mean arterial pressure (MAP), temperature, oxygen saturation $(\mathrm{SpO} 2)$, intracranial pressure (ICP), cerebral perfusion pressure (CPP), respiratory rate, and ventricular drain output) from the first 3 days of admission were collected. Hourly ICP and CPP values were obtained, and other physiologic values were available at a resolution of every 1 to $4 \mathrm{~h}$. For each predictor, we computed the minimum, maximum, median, and variance for the first 1, 2, 3, 1-2, 2-3, and 1-3 days.

Two neurologists independently abstracted and adjudicated the presence of DCI following a previously published protocol, with excellent inter-rater agreement $(95.83 \%)$ [12]. DCI was defined using published guidelines [13].

We excluded duplicate features from analysis and variables that served as surrogates of our outcome measure, e.g., discharge disposition, and duration of hospitalization. Variables with greater than $10 \%$ missing data were discarded, and missing values were imputed for the rest.

\section{Descriptive Statistics}

Mean, median, and inter-quartile ranges were calculated for descriptive analysis. Univariate analysis was performed using a linear regression model, and significance was set at $<0.05$. 


\section{Predictive Modeling: Model Estimation and Validation}

In this big data study, we performed predictive, instead of explanatory modeling, to predict new or future observations. Determining causality is not the primary goal nor a prerequisite for inclusion of a variable in a predictive model [14]. Complex and potentially uninvestigated associations can be used to generate new hypotheses in predictive modeling. This can potentially improve and provide further pathophysiologic understanding of existing explanatory models [14].

We created predictive models and estimated their performance using nested cross-validation (CV) [15]. Details are provided in supplementary material. In summary, our CV approach included four features:

1. Dividing data into testing and training sets (external $C V$ ) We used a tenfold external CV to validate model performance. $10 \%$ of the available data were held out, while the remaining $90 \%$ were used for model optimization (feature selection and parameter tuning).

2. Balancing the training data to cope with class imbalance To prevent bias toward predicting the predominant outcome, in each fold of external CV we balanced the training data by randomly discarding examples from the dominant class(es) until all classes had equal numbers of examples.

3. Inner cross-validation for model optimization For each round of external $\mathrm{CV}$, we conducted an inner CV loop for feature selection and to identify the optimal value of the penalty parameter $\lambda$. The level of complexity $(\lambda)$ that produced the best performance on the internal testing data was then used to train a predictive model on all the training data.

4. Model evaluation For each round of external CV, we tested the logistic regression model developed in the inner CV loop on the held out testing data. Model performance was assessed using the area under the receiver operating curve (AUROC). Ten AUROC values were obtained for each round of CV. The final reported predictive performance is the mean and standard deviation of the AUROC across the tenfold of CV. This ensured that performance estimates are based entirely on data not used for feature selection or model parameter tuning, avoiding reporting prediction results that are inflated by model overfitting. Conventional approaches that do not enforce strict separation between training and testing data (e.g., fitting a predictive model that includes features with small $p$ values on univariate analysis) are vulnerable to overfitting.

Steps 1-4 were repeated 1000 times to obtain final performance estimates. Each round of bootstrapping involved a different random subset of the available data, yielding different sets of optimal features. We therefore report not a single set of features, but rather the frequency with which features were selected. This more accurately estimates the robustness of each feature for outcome prediction that can be obtained from a single round of tenfold $\mathrm{CV}$.

\section{Binary and Multilevel Outcome Prediction Models}

We created two types of models using the nested-CV framework. First we created a binary prediction algorithm for predicting in-hospital mortality (GOS 1 vs GOS 2-5) and one for predicting death/dependence versus independence (GOS 1-3 vs GOS 4-5). Second, we created a multiclass prediction algorithm, which predicted poor (GOS 1-2), intermediate (GOS 3), and good (GOS 4-5) outcomes.

All statistical analyses were performed using MATLAB version 2016a (Natick, MA).

\section{Results}

\section{Cohort Characteristics}

Of 209 medical charts reviewed, 56 were excluded due to the absence of aneurysm, or clear alternate etiology, and 153 subjects were included. Demographic and clinical variables are summarized in Table 1 . The mean age was 58.3 years, and $69.3 \%(n=106)$ were female. The mean APACHE II score was 14.4. Most patients presented with Hunt and Hess $(\mathrm{HH}) 4(n=39,25.5 \%)$ and Fisher 3 $(n=114,74.5 \%)$ hemorrhages. $47.7 \%(n=73)$ aneurysms were coiled, and $41.8 \%(n=64)$ were clipped. Majority of patients had a discharge GOS of 3. Twentyeight (18\%) patients died in the hospital, 27 following withdrawal of life-sustaining therapies, and one met brain death criteria. A total of 138 patients underwent EEG monitoring; epileptiform discharges $(n=65,47.1 \%)$ and rhythmic delta activity $(n=60,43.4 \%)$ were the most frequent abnormalities. Eleven patients had depth EEG monitoring. Of 473 defined candidate predictor variables, 22 were excluded from analysis due to missing values.

\section{Binary Discrimination: Predictors of Mortality}

Significant predictors of mortality on univariate analysis are shown in Fig. 1. These included total number of AEDs, levetiracetam and lorazepam, APACHE II score, aneurysm treatment modality, periodic discharges, and HH Score.

The main predictors of death at discharge in the multivariate model, and the frequency of selection in the bootstrapping method are shown in Table 2. APACHE II and glucose and ICP variance were selected more than $95 \%$ 
Table 1 Clinical and demographic variables

\begin{tabular}{lll}
\hline Age: mean (SD) & 58.3 & $(14.2)$ \\
Gender & Female & \\
Frequency (\%) & 106 & $(69.3 \%)$ \\
GCS on admission, mean (SD) & 10.4 & $(4.7)$ \\
APACHE II on admission, mean (SD) & 14.4 & $(7.6)$ \\
Hunt and Hess score, frequency (\%) & & \\
1 & 32 & $(20.9 \%)$ \\
2 & 31 & $(20.3 \%)$ \\
3 & 30 & $(19.6 \%)$ \\
4 & 39 & $(25.5 \%)$ \\
5 & 21 & $(13.7 \%)$
\end{tabular}

Fisher grade, frequency $(\%)$

1

2

3

4

Mode of aneurysm treatment, frequency (\%)

Coil

Clip

Coil + clip

Flow diverter

Flow diverter + coil

Untreated (death prior to treatment)

Rebleed: frequency (\%)

Delayed cerebral ischemia: frequency $(\%)$

Mechanical ventilation: frequency $(\%)$

Duration of mechanical ventilation in days, mean (SD)

Duration of ICU stay in days, mean (SD)

Duration of hospital stay in days, mean (SD)

Clinical seizures at initial presentation: frequency, $(\%)$ ( $<24 \mathrm{~h}$ from ictus)

Clinical seizures during hospitalization: frequency, $(\%)$ ( $>24 \mathrm{~h}$ from ictus)

Scalp EEG findings (138 patients monitored)

Periodic discharges: frequency $(\%)$

Rhythmic delta activity: frequency (\%)

Epileptiform discharges: frequency $(\%)$

Seizures: frequency $(\%)$

Depth EEG findings ( 9 patients monitored)

Seizures and periodic patterns: frequency $(\%)$

Patients on at least one AED throughout hospitalization, frequency (\%)

HAI frequency (\%)

Pneumonia

Urinary tract infection

Meningitis/ventriculitis

Bacteremia/sepsis

All HAIs combined

1

$(7.20 \%)$

$114 \quad(74.5 \%)$

27

(17.6\%)

73

(47.7\%)

64

(41.8\%)

3

5

(2.00\%)

$(3.30 \%)$

$(0.65 \%)$

(4.60\%)

(8.5)

13

68

99

(44.4\%)

$(64.7 \%)$

5.50

(7.3)

14.6

(7.4)

18.0

(9.3)

22

(14.4\%)

9

$(5.9 \%)$

$21 \quad(15.2 \%)$

$60 \quad(43.4 \%)$

$65 \quad(47.1 \%)$

$6 \quad(4.30 \%)$

$6 \quad(6.5 \%)$

$53 \quad(34.6 \%)$

$56 \quad(36.6 \%)$

$35 \quad(20.9 \%)$

$5 \quad(3.27 \%)$

$2 \quad(1.30 \%)$

$81 \quad(52.9 \%)$

GOS at discharge, frequency (\%)

1

2

3

4

$28 \quad(18.3 \%)$

$3 \quad(2.00 \%)$

$94 \quad(61.4 \%)$

$20 \quad(13.1 \%)$

$8 \quad(5.23 \%)$

Discharge location, frequency (\%)

Home

$38 \quad(24.8 \%)$

Rehab/skilled nursing facility/long-term acute care

87

$(56.9 \%)$

Frequency depicts number of patients (n); AED anti-epileptic drug, APACHE II acute physiology and chronic health evaluation II, EEG electroencephalogram, GCS Glasgow Coma Scale, GOS Glasgow Outcome Scale, HAI healthcare-associated infections, $S D$ standard deviation 
of the time. At the point of maximum accuracy on the ROC curve, the sensitivity was $86 \%$, and specificity was $92 \%$; the mean AUC was 0.9198. Performance metrics and ROC curve are shown in supplementary material (Figure e-1 and e-2).

\section{Binary Discrimination: Predictors of Death/ Dependence Versus Independent Status}

Significant predictors of death/dependence (GOS 1-3) versus independent status (GOS 4-5) on univariate analysis are shown in Fig. 2. These included total number of AEDs, levetiracetam, APACH II, admission GCS, and HH score. Epileptiform discharges and rhythmic delta activity were significant EEG findings. The presence of HAIs and hospital-acquired pneumonia were also significant.

Table 2 shows the features selected in the multivariate model. Levetiracetam and mechanical ventilation were selected in a $100 \%$ of the training sessions. The sensitivity and specificity at the point of maximum accuracy on the ROC curve were 94 and 98\%, respectively, and the mean
AUC was 0.9456. Performance metrics and ROC curve are shown in supplementary material (Figure e-3 and e-4).

\section{Multilevel Discrimination: Predictors of Poor, Intermediate, or Good Outcomes}

Predictors of outcome in the multivariate multilevel prediction model are shown in Table 2. Maximum day 1 GCS, minimum day 2-3 GCS, and APACHE II score were the most frequently selected features. Periodic discharges, lacosamide, and rebleed were less frequently selected. Using these features, the model predicted poor and good outcomes with greater than $80 \%$ accuracy and intermediate outcome with greater than $70 \%$ accuracy (Fig. 3).

\section{Discussion}

In this large EHR data-driven predictive model, we identified key features that accurately predicted outcomes in patients with aSAH. Our study highlights the importance of

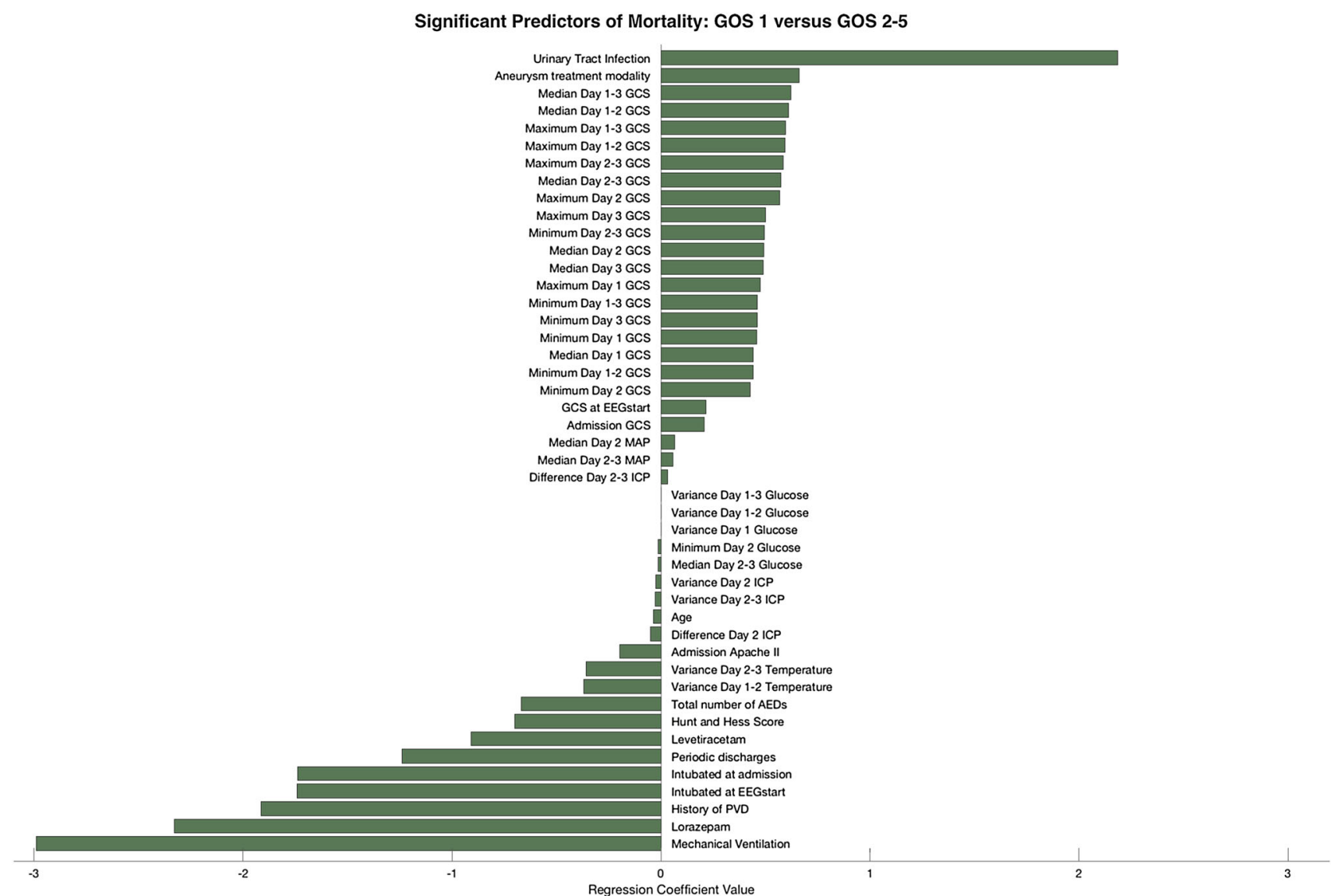

Fig. 1 Significant predictors of mortality on univariate analysis: GOS 1 versus GOS 2-5. Significant predictors of outcome on univariate analysis and their regression coefficients are shown. The $p$ value for each predictor was $<0.05$. For predictors with negative regression coefficient, presence/higher value was associated with lower GOS. For predictors with positive regression coefficient, presence/higher value was associated with higher GOS 
Table 2 Predictors of outcome on multivariate analysis

Binary discrimination: predictors of mortality (GOS 1 vs GOS 2-5)

99\% of training sessions: variance in day 3 glucose

98\% of training sessions: admission APACHE II

$98 \%$ of training sessions: variance in day 2 ICP

95\% of training sessions: variance in day 1 glucose

$92 \%$ of training sessions: maximum day 1 GCS

$89 \%$ of training sessions: median day $3 \mathrm{WBC}$ count

$86 \%$ of training sessions: minimum day $1-3 \mathrm{SpO} 2$

$68 \%$ of training sessions: maximum day $1-3$ sodium

$51 \%$ of training sessions: maximum day 2 MAP

$17 \%$ of training sessions: variance in day 3 sodium

$12 \%$ of training sessions: difference in day 3 sodium

$1 \%$ of training sessions: maximum day $2-3$ GCS

Binary discrimination: predictors of death/dependence versus independent status (GOS 1-3 vs GOS 4-5)

$100 \%$ of training sessions: levetiracetam

$100 \%$ of training sessions: mechanical ventilation

$99 \%$ of training sessions: duration of mechanical ventilation

94\% of training sessions: minimum day $2-3 \mathrm{WBC}$ count

$87 \%$ of training sessions: maximum day $1-3$ heart rate

$85 \%$ of training sessions: variance in day 2 ICP

$78 \%$ of training sessions: difference day $2-3$ GCS

$73 \%$ of training sessions: median day 1 GCS

$54 \%$ of training sessions: age

$54 \%$ of training sessions: maximum day 1 external ventricular drainage

$48 \%$ of training sessions: variance in day 2 sodium

$33 \%$ of training sessions: epileptiform discharges

$22 \%$ of training sessions: total no of AEDs

$22 \%$ of training sessions: difference day 1 temperature

$16 \%$ of training sessions: maximum day $2 \mathrm{WBC}$

$4 \%$ of training sessions: Admission APACHE II score

$2 \%$ of training sessions: DCI

$1 \%$ of training sessions: difference day 3 MAP

Multiclass discrimination: predictors of poor versus intermediate versus good outcomes (GOS 1-2 vs GOS 3 vs GOS 4-5)

$100 \%$ of training sessions: maximum day $1 \mathrm{GCS}$

$97 \%$ of training sessions: minimum day 2-3 GCS

$90 \%$ of training sessions: Admission APACHE II

$6 \%$ of training sessions: median day $1-3$ GCS

$3 \%$ of training sessions: maximum day $1-3$ GCS

$1 \%$ of training sessions: periodic discharges

$1 \%$ of training sessions: maximum day $2-3$ external ventricular drainage

$1 \%$ of training sessions: lacosamide

$1 \%$ of training sessions: rebleed

The frequency with which each variable was selected in the bootstrapping method is shown. $A E D$ anti-epileptic drugs, $D C I$ delayed cerebral ischemia, GCS Glasgow Coma Scale, GOS Glasgow Outcome Scale, ICP intracranial pressure, MAP mean arterial pressure; $S p O 2$ pulse oxygen saturation, $W B C$ white blood cell

fluctuations and variance in physiologic features, which often more accurately predict outcome than the minimum or maximum value. The predictors identified do not necessarily imply causality. Nevertheless, some of the identified associations suggest plausible causal hypotheses and potentially modifiable risk factors and warrant further investigation in prospective studies. 
Significant Predictors of Death/Dependance versus Indenepenent Status: Gos 1-3 versus GOS 4-5

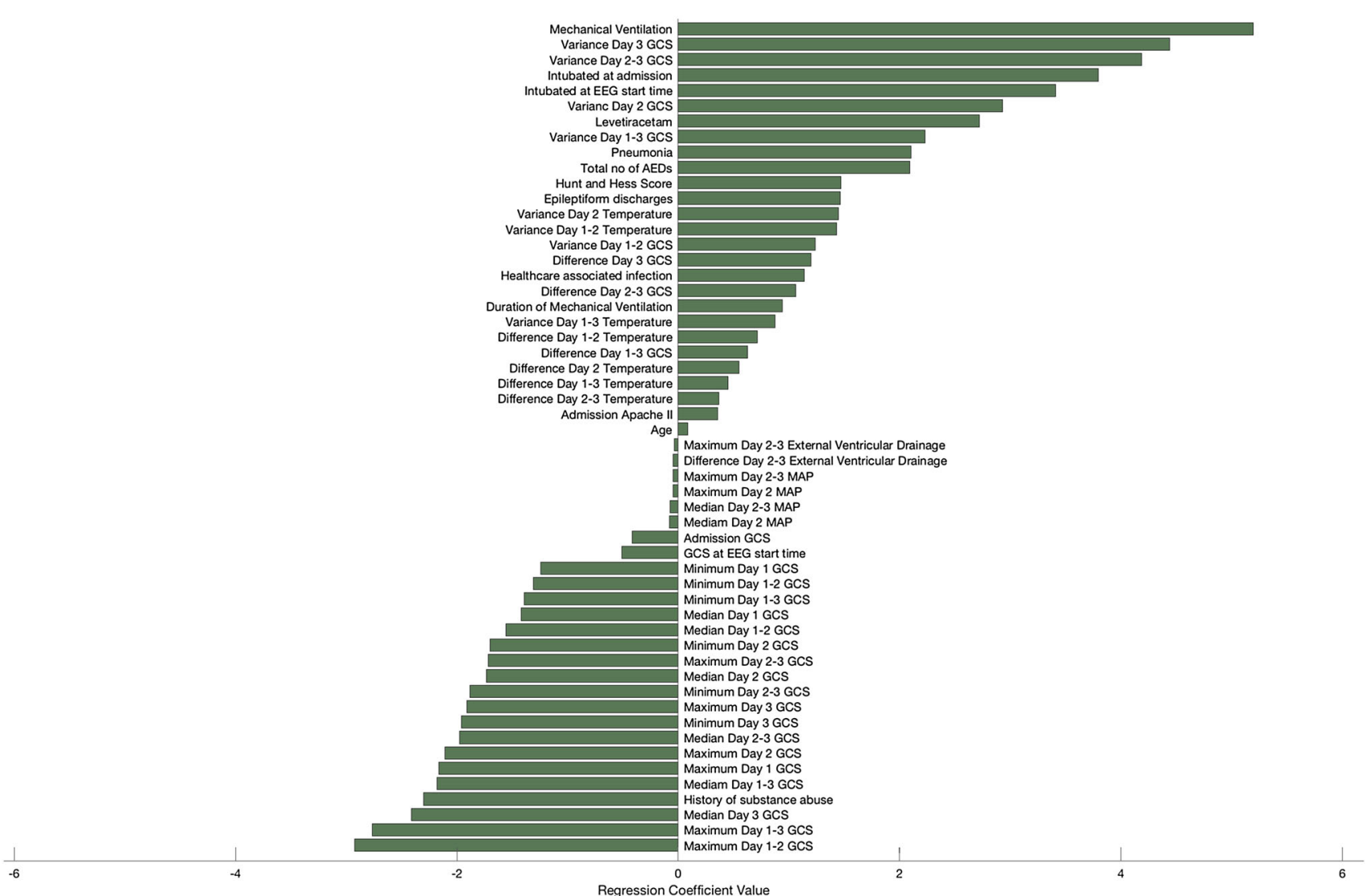

Fig. 2 Significant predictors of death/dependence versus independent status on univariate analysis: poor (GOS 1-3) versus (GOS 4-5). Significant predictors of outcome on univariate analysis and their regression coefficients are shown. The $p$ value for each predictor was $<0.05$. For predictors with positive regression coefficient,

aSAH hospital mortality rate is reported at 20-50\% [16], with up to $40 \%$ of deaths from extra-cerebral organ failure [2]. Our $18 \%$ in-hospital mortality is comparable to that of the Columbia University SAH Outcomes Project [16]. The APACHE II score consistently predicted mortality. APACHE II is a disease severity score incorporating physiologic and laboratory data [17]. The physiological component is also included in the FRESH score [5]. Looking beyond the individual components of the APACHE II, we identified ICP, external ventricular drain (EVD) drainage, EEG findings as additional predictors. Apart from GCS, the APACHE II does not include physiologic or clinical data that are more specific to the nervous system; hence, addition of these factors can enhance performance of predictive scores.

Variance, maximum, and absolute difference in serum sodium levels predicted outcomes. Sodium derangements correlate with death and disability $[2,16,18]$, and fluctuations may have a greater impact on outcomes than hyponatremia itself $[18,19]$. Strict sodium control and presence/higher value was associated with lower GOS. For predictors with negative regression coefficient, presence/higher value was associated with higher GOS. EEG: Electroencephalogram; GCS: Glasgow Coma Scale; MAP: mean arterial pressure; PVD: peripheral vascular disease

balancing the effects of salt wasting, syndrome of inappropriate $\mathrm{ADH}$, and hyperosmolar treatment may mitigate the adverse effects. Similarly, serum glucose derangements can increase secondary cerebral injury [20, 21]. While there is conflicting data on the impact of tight glycemic control, similar to prior studies, our findings suggest glucose variability correlates with outcomes in patients with neurological injury [20,21].

Other laboratory predictors included minimum and maximum WBC count. Leukocytosis has been identified as a predictor of poor outcome in SAH [22, 23] and also as an independent predictor of vasospasm [24]. A rising WBC count warrants vigilance and should raise suspicion for potential vasospasm.

Cardiac and pulmonary complications occur in up to 63 and $80 \%$ of aSAH patients, respectively [2]. Blood pressure extremes and heart rate variability can impact outcomes $[6,25,26]$. We found maximum heart rate and maximum MAP predicted outcomes. Pulmonary predictors included duration of mechanical ventilation and minimum $\mathrm{SpO} 2$. 


\section{Predicted versus Observed Outcomes}

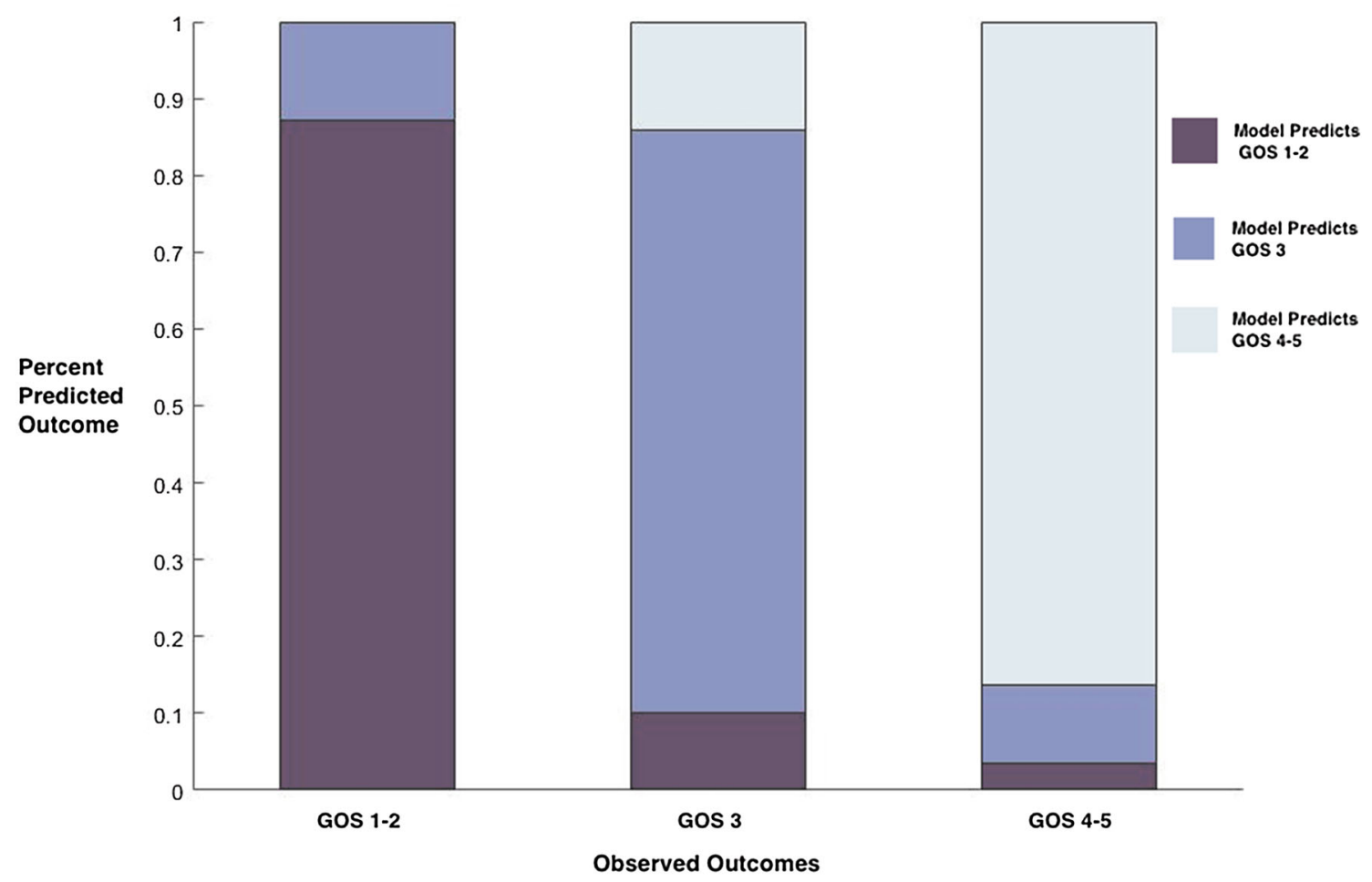

Fig. 3 Multiclass model-predicted versus observed outcomes; poor (GOS 1-2), intermediate (GOS 3), good (GOS 4-5). The percentage of accurately predicted discharge GOS is shown. The first stacked bar

These predictors could certainly be surrogates of the underlying disease severity. Alternatively, they may directly impact outcomes; for example in patients without lung injury, who require ventilation for depressed arousal, over ventilation can result in hyperoxia or hypocapnea. Oxidative stress may exacerbate cerebral injury and increase the risk of DCI [27]. Hypocapnea is similarly associated with worse outcomes and vasospasm [28]. These potential modifiable clinical factors can be addressed using a protocolized approach to ventilator management.

Temperature differences also predicted outcomes, and although HAIs have been associated with worse outcomes $[2,29]$, and were significant in our univariate analysis, they were not identified in our multivariate model. One hypothesis is that earlier physiologic derangements may have greater impact on outcomes.

We also investigated ICP, CPP, and EVD drainage and found ICP variance to be a significant predictor. Data on ICP monitoring in aSAH are limited [30] with widespread practice variation [31]. Elevated ICP is linked to worse outcomes, although this may be a reflection of the disease severity [32], and ICP-derived variables, such as pressure reactivity index and variability, may be more accurate predictors [30]. There is also conflicting data on optimal EVD management [33-35]. Continuous drainage may be shows the model accurately predicts poor outcome (GOS 1-2) $87 \%$ of the time. The second bar shows the model accurately predicts an intermediate outcome (GOS 3) $74 \%$ of the time

associated with greater risk of infection but lower risk of vasospasm [33, 34]. We typically use intermittent drainage, transitioning to continuous drainage if clinically indicated. Five of our patients developed meningitis, though this was not identified as an outcome predictor. Simultaneously, avoiding prolonged weans and continuous drainage when not indicated may help prevent EVD-related complications.

Age, GCS, and rebleeding were significant predictors that are also incorporated in existing scores $[1,5]$, although rebleeding was only seen in $1 \%$ of training sessions. Interestingly, $\mathrm{HH}$ score was identified only on univariate and not on multivariate analysis, underscoring the potential advantages of scores that are not limited to the initial examination.

Forty four percent of our patients had DCI, higher than typical rates cited in the literature (up to 30\%) [13]. This may be because majority of our patients were high grade. Although not identified as a predictor of death, the presence of DCI did help discriminate between dependent versus independent status at discharge.

Finally, we studied the impact of EEG features. Epileptiform and periodic discharges were predictors in the multiclass model. Inter-ictal patterns are associated with worse outcomes and DCI, potentially related to increased cerebral blood flow or metabolism [36-38]. Larger 
prospective studies can determine the long-term impact of periodic patterns and implications of treatment. Careful selection of patients for treatment using existing evidence may limit both the impact of these patterns and avoid excess use of AEDs.

Electrographic seizures, seen only in 6 of our patients, were not identified in our model, despite prior studies showing an association with worse outcomes [39]. Our findings raise the hypothesis that inter-ictal scalp activity may signify scalp-negative seizures [40], or seizures, per se, may fail to predict outcome if treated in a timely fashion. Intriguingly, we found that use of AEDs predicted poor outcomes. This association is not necessarily causal. There are two plausible explanations for this (Figure e-5 supplementary material); AEDs are a non-causal predictive variable, but have an "apparent causality" as they are surrogates for the underlying disease severity or EEG findings (confounding by indication). Alternatively, AEDs may have an iatrogenic, true-negative causal impact on outcomes. Regardless, we hypothesize that prompt discontinuation of primary AED prophylaxis, and using the lowest dose of monotherapy for secondary prophylaxis, might be beneficial.

Limitations of our study are its retrospective nature and that it is a single-center study. Only including patients with a definite aneurysm resulted in a smaller sample size. Most patients were high-grade aSAH, limiting the generalizability of our findings to lower-grade hemorrhages, and our inclusion of intraventricular hemorrhage was limited to the Fisher score. Variation in AED prescribing practices for secondary prophylaxis and temporal trends in aneurysm treatment modality are additional limitations. The greatest difference in aneurysm treatment, however, was in the first year (2012: $23 \%$ coiled, $76 \%$ clipped); thereafter, there was an increase in coiling (2013: 50\% coiled, 46.9\% clipped; 2014: $39 \%$ coiled, 50\% clipped; 2015: $57.1 \%$ coiled, 26.8\% clipped; 2016: 58\% coiled, $25 \%$ clipped).

\section{Conclusions}

Attractive features of data-driven approaches to outcome prediction are reproducibility, lack of susceptibility to errors of human judgment, and their ability to take advantage of non-obvious patterns in complex medical data. In our cohort, variability and fluctuations in physiological and laboratory data were important predictors of outcomes that are not readily available in a clinician-documented approach. Early identification of these features may identify patients requiring additional vigilance and facilitate more timely therapeutic interventions, allowing for improved immediate outcomes and rehabilitation potential. Future prospective studies are needed to create a more comprehensive, reliable, and reproducible outcome prediction score using additional features such as ICP, blood glucose levels, EEG findings, and variability in physiologic data. With increasing use of multimodality monitoring, larger prospective studies can better understand the relationship between variability in physiologic and laboratory data and cerebral metabolism, and the impact of goal-directed treatments on neurologic outcomes.

Acknowledgements This study was supported by NIH-NINDS (1K23NS090900) and the Andrew David Heitman Foundation.

Authors' Contributions SFZ and EN conceptualized and designed the study, performed data collection and management, performed analysis, and drafted the original, revised, and final manuscript. SB performed data collection and management and data analysis and reviewed and revised the manuscript. LF performed data analysis and reviewed and revised the manuscript. EJB, SB, KO', AS, and DJ performed data collection and reviewed and revised the manuscript. $\mathrm{JK}, \mathrm{MS}$, and ABP reviewed and revised the manuscript. ESR conceptualized and designed the study and reviewed and revised the manuscript. MBW conceptualized and designed the study, performed analysis, and reviewed and revised the manuscript.

\section{Compliance with Ethical Standards}

Conflict of interest Sahar F. Zafar receives research support from an institutional contract with SAGE therapeutics. Jennifer Kim receives funding from NIH-NINDS R25 NS065743. Aman B. Patel is a consultant for Medtronic and Penumbra. Eric $S$ Rosenthal receives research support from an institutional contract with SAGE therapeutics and received funding for this work from the Andrew David Heitman Foundation. M. Brandon Westover receives funding from NIH-NINDS 1K23NS090900 and the Andrew David Heitman Foundation. Eva N Postma, Siddharth Biswal, Lucas Fleuren, Emily J Boyle, Sophia Bechek, Kathryn O'Connor, Apeksha Shenoy, Durga Jonnalagadda, and Mouhsin Shafi report no disclosures.

\section{References}

1. Lee VH, Ouyang B, John S, et al. Risk stratification for the inhospital mortality in subarachnoid hemorrhage: the HAIR score. Neurocrit Care. 2014;1(21):14-9.

2. Stevens RD, Nyquist PA. The systemic implications of aneurysmal subarachnoid hemorrhage. J Neurol Sci. 2007;261:143-56.

3. Fisher CM, Kistler JP, Davis JM. Relation of cerebral vasospasm to subarachnoid hemorrhage visualized by computerized tomographic scanning. Neurosurgery. 1980;6(1):1-9.

4. Teasdale GM, Drake CG, Hunt W, et al. A universal subarachnoid hemorrhage scale: report of a committee of the World Federation of Neurosurgical Societies. J Neurol Neurosurg Psychiatry. 1988;51(11):1457.

5. Witsch J, Frey HP, Patel S, et al. Prognostication of long-term outcomes after subarachnoid hemorrhage: the FRESH score. Ann Neurol. 2016;80:46-58.

6. Claassen J, Vu A, Kreiter KT, et al. Effect of acute physiologic derangements on outcome after subarachnoid hemorrhage. Crit Care Med. 2004;32:832-8. 
7. Jennett B, Bond M. Assessment of outcome after severe brain damage: a practical scale. The Lancet. 1975;305:480-4.

8. Agrawal D, Joshua SP, Gupta D, Sinha S, Satyarthee GD. Can Glasgow score at discharge represent final outcome in severe head injury? J Emerg Trauma Shock. 2012;5:217.

9. Lemcke J, Ahmadi S, Meier U. Outcome of patients with severe head injury after decompressive craniectomy. Acta Neurochir Suppl. 2010;106:231-3. doi:10.1007/978-3-211-98811-4_43.

10. De Guise E, LeBlanc J, Feyz M, Lamoureux J. Prediction of outcome at discharge from acute care following traumatic brain injury. J Head Trauma Rehabil. 2006;21:527-36.

11. Hirsch LJ, LaRoche SM, Gaspard N, Gerard E, Svoronos A, Herman ST, Mani R, Arif H, Jette N, Minazad Y, Kerrigan JF. American Clinical Neurophysiology Society's standardized critical care EEG terminology: 2012 version. J Clin Neurophysiol. 2013;30(1):1-27.

12. Zafar SF, Westover MB, Gaspard N, et al. Interrater agreement for consensus definitions of delayed ischemic events after aneurysmal subarachnoid hemorrhage. J Clin Neurophysiol. 2016;33:235-40.

13. Vergouwen MD, Vermeulen M, van Gijn J, et al. Definition of delayed cerebral ischemia after aneurysmal subarachnoid hemorrhage as an outcome event in clinical trials and observational studies. Stroke. 2010;41:2391-5.

14. Waljee AK, Higgins PD, Singal AG. A primer on predictive models. Clin Trans Gastroenterol. 2014;5(1):e44.

15. Friedman J, Hastie T, Tibshirani R. The elements of statistical learning, vol. 1., Springer series in statisticsBerlin: Springer; 2001.

16. Lantigua H, Ortega-Gutierrez S, Schmidt JM, et al. Subarachnoid hemorrhage: who dies, and why? Crit Care. 2015;19:309.

17. Knaus WA, Draper EA, Wagner DP, Zimmerman JE. APACHE II: a severity of disease classification system. Crit Care Med. 1985;13:818-29.

18. Bales J, Cho S, Tran TK, et al. The effect of hyponatremia and sodium variability on outcomes in adults with aneurysmal subarachnoid hemorrhage. World Neurosurg. 2016;96:340-9.

19. Okazaki T, Hifumi T, Kawakita K, et al. 783: sodium variability is an independent predictor of neurologic outcomes in subarachnoid hemorrhage. Crit Care Med. 2016;44:272.

20. Okazaki T, Hifumi T, Kawakita K, et al. Blood glucose variability: a strong independent predictor of neurological outcomes in aneurysmal subarachnoid hemorrhage. J Intensive Care Med. 2016. doi: $10.1177 / 0885066616669328$.

21. Kurtz P, Claassen J, Helbok R, et al. Systemic glucose variability predicts cerebral metabolic distress and mortality after subarachnoid hemorrhage: a retrospective observational study. Crit Care. 2014;18:R89.

22. Tam AK, Ilodigwe D, Mocco J, et al. Impact of systemic inflammatory response syndrome on vasospasm, cerebral infarction, and outcome after subarachnoid hemorrhage: exploratory analysis of CONSCIOUS-1 database. Neurocrit Care. 2010;13:182-9.

23. Szklener S, Melges A, Korchut A, et al. Predictive model for patients with poor-grade subarachnoid haemorrhage in 30-day observation: a 9-year cohort study. BMJ Open. 2015;5:e007795.

24. McGirt MJ, Mavropoulos JC, McGirt LY, et al. Leukocytosis as an independent risk factor for cerebral vasospasm following aneurysmal subarachnoid hemorrhage. J Neurosurg. 2003;98:1222-6.

25. Degos V, Apfel CC, Sanchez P, et al. An admission bioclinical score to predict 1-year outcomes in patients undergoing aneurysm coiling. Stroke. 2012;43:1253-9.

26. Schmidt JM, Sow D, Crimmins M, et al. Heart rate variability for preclinical detection of secondary complications after subarachnoid hemorrhage. Neurocrit Care. 2014;20:382-9.

27. Jeon SB, Choi HA, Badjatia N, et al. Hyperoxia may be related to delayed cerebral ischemia and poor outcome after subarachnoid haemorrhage. J Neurol Neurosurg Psychiatry. 2014;85:1301-7.

28. Solaiman O, Singh JM. Hypocapnia in aneurysmal subarachnoid hemorrhage: incidence and association with poor clinical outcomes. J Neurosurg Anesthesiol. 2013;25:254-61.

29. Douds GL, Tadzong B, Agarwal AD, Krishnamurthy S, Lehman EB, Cockroft KM. Influence of fever and hospital-acquired infection on the incidence of delayed neurological deficit and poor outcome after aneurysmal subarachnoid hemorrhage. Neurol Res Int. 2012;2012:6.

30. Cossu G, Messerer M, Stocchetti N, Levivier M, Daniel RT, Oddo M. Intracranial pressure and outcome in critically ill patients with aneurysmal subarachnoid hemorrhage: a systematic review. Minerva Anestesiol. 2016;82:684-96.

31. Mack WJ, King RG, Ducruet AF, et al. Intracranial pressure following aneurysmal subarachnoid hemorrhage: monitoring practices and outcome data. Neurosurg Focus. 2003;14:1-5.

32. Heuer GG, Smith MJ, Elliott JP, Winn HR, LeRoux PD. Relationship between intracranial pressure and other clinical variables in patients with aneurysmal subarachnoid hemorrhage. J Neurosurg. 2004;101:408-16.

33. Olson DM, Zomorodi M, Britz GW, Zomorodi AR, Amato A, Graffagnino C. Continuous cerebral spinal fluid drainage associated with complications in patients admitted with subarachnoid hemorrhage: clinical article. J Neurosurg. 2013;119:974-80.

34. Qian C, Yu X, Chen J, et al. Effect of the drainage of cerebrospinal fluid in patients with aneurismal subarachnoid hemorrhage: A meta-analysis. Medicine. 2016;95(41):e5140.

35. Klopfenstein JD, Kim LJ, Feiz-Erfan I, et al. Comparison of rapid and gradual weaning from external ventricular drainage in patients with aneurysmal subarachnoid hemorrhage: a prospective randomized trial. J Neurosurg. 2004;100:225-9.

36. Foreman B, Claassen J. Quantitative EEG for the detection of brain ischemia. Crit Care. 2012;16:216.

37. Claassen J, Hirsch LJ, Frontera JA, et al. Prognostic significance of continuous EEG monitoring in patients with poor-grade subarachnoid hemorrhage. Neurocrit Care. 2006;4:103-12.

38. Struck AF, Westover MB, Hall LT, Deck GM, Cole AJ, Rosenthal ES. Metabolic correlates of the ictal-interictal continuum: FDG-PET during continuous EEG. Neurocrit Care. 2016;24:324-31.

39. De Marchis GM, Pugin D, Meyers E, et al. Seizure burden in subarachnoid hemorrhage associated with functional and cognitive outcome. Neurology. 2016;86:253-60.

40. Mikell CB, Dyster TG, Claassen J. Invasive seizure monitoring in the critically-Ill brain injury patient: current practices and a review of the literature. Seizure. 2016;41:201-5. 